\title{
PCA sets and convexity
}

\author{
by
}

Robert Ka ufman (Urbana, IL)

\begin{abstract}
Three sets occurring in functional analysis are shown to be of class PCA (also called $\Sigma_{2}^{1}$ ) and to be exactly of that class. The definition of each set is close to the usual objects of modern analysis, but some subtlety causes the sets to have a greater complexity than expected. Recent work in a similar direction is in $[1,2,10,11,12]$.
\end{abstract}

I. Extreme points and integrals. Suppose that $S$ is a subset of a linear space; a classical problem in analysis concerns representation of elements of $S$ by integrals over extreme points of $S$. The work of Choquet and Bishop-de Leeuw [13] relies on compactness; the theorem of Edgar [8, 6] relies on geometric properties of the space containing $S$ and on set-theoretic ideas. Our interest is in the set $\iota(\operatorname{ex} K)$ of elements which can be represented by an integral over ex $K$, where $K$ is closed, bounded, convex, and separable. More generally, let $\mathcal{S}$ be a co-analytic set in $K$; then $y \in \iota(\mathcal{S})$ means that $y=\int x d \mu(x)$, where $\mu$ is a probability measure in $K$ such that $\mu^{*}(S)=1$. (We write $\mu^{*}(\mathcal{S})$ because $S$ need not be a Borel set.)

Theorem 1. The set $\iota(\mathcal{S})$ is a PCA set.

Theorem 2. For each PCA set $\Sigma$ there is a closed, bounded, convex set $K$ in $c_{0}$ such that $\Sigma$ is homeomorphic to a closed subset of $\iota(\operatorname{ex} K)$.

Proof of Theorem 1. We denote by $P^{*}(\mathcal{S})$ the set of probability measures occurring in the definition of $\iota(\mathcal{S})$. Let $K_{1}$ be a compact metric space containing $K$ as a $G_{\delta}$, so that $K_{1} \backslash \mathcal{S}$ is an analytic set in $K_{1}$. Thus $K_{1} \backslash \mathcal{S}=h(V)$, where $h$ is a continuous function on some $G_{\delta}$-set $V$. By a small adjustment we can assume that $h$ is continuous on a compact metric space $Y_{1} \supseteq V$ and that $h\left(Y_{1}\right)=K_{1}$.

Lemma 1. $P^{*}(\mathcal{S})$ is a co-analytic set in $P\left(K_{1}\right)$.

2000 Mathematics Subject Classification: Primary 03E15, 54H05; Secondary 46A55, $46 \mathrm{~B} 03$. 
Proof. Let $M^{+}(V)$ be the set of probability measures $\mu$ in $Y_{1}$ such that $\mu(V)>0$. If $\mu \in M^{+}(V)$ then $\left(h^{*} \mu\right)\left(K_{1} \backslash \mathcal{S}\right) \geq \mu(V)>0$ whence $h^{*} \mu \notin P^{*}(\mathcal{S})$. Conversely, if $\lambda \notin P^{*}(\mathcal{S})$ and $\lambda\left(K_{1}\right)=1$, then there is a compact set $Y_{2} \subseteq V$ such that $\lambda\left(h\left(Y_{2}\right)\right)>\frac{1}{2} \lambda\left(K_{1} \backslash \mathcal{S}\right)>0$. Thus it is easy to see that $\lambda=h^{*} \mu$ for some $\mu$ such that $\mu\left(Y_{2}\right)>0$ and so $\mu(V)>0$. Thus $P^{*}(\mathcal{S})$ is the complement of $h^{*}\left(M^{+}(V)\right)$. But $M^{+}(V)$ is a Borel set, in fact a set of type $G_{\delta \sigma}$, whence $h^{*}\left(M^{+}(V)\right)$ is analytic and $P^{*}(\mathcal{S})$ is co-analytic.

Let $\left(x_{m}^{*}\right)_{n=1}^{\infty}$ be a total sequence of bounded linear functionals on the B-space containing $K$, and let $K_{1}$ be defined so that the functionals $x_{m}^{*}$ can be extended continuously over $K_{1}$. Then $x \in \iota(\mathcal{S})$ if and only if there is a measure $\mu \in P^{*}(\mathcal{S})$ such that $x_{m}^{*}(x)=\int x_{m}^{*} d \mu$ for $m \geq 1$; from this it follows that $\iota(\mathcal{S})$ is a PCA set.

Proof of Theorem 2. Let $\Sigma=p(\mathcal{S})$ where $\mathcal{S}$ is a co-analytic set in a compact metric space $X_{1}$ (and $p$ is continuous on $X_{1}$ ). Now $[10,12]$ there is a closed, bounded, convex set $K_{0}$ in $P\left(X_{1}\right) \oplus c_{0}$ such that ex $K_{0}$ is just the set of points $\left(\delta_{y}, 0\right)$ with $y \in \mathcal{S}$. Here $P\left(X_{1}\right)$ is realized as a compact subset of $c_{0}$, so that $K_{0} \subseteq c_{0} \oplus c_{0}$. A similar observation applies to the set $M_{1}\left(X_{1}\right)$ of measures on $X_{1}$ of total variation at most $1 . X_{2}$ is defined to be $p\left(X_{1}\right)$, so that $X_{2} \supseteq \Sigma$.

Let $x \in c_{0}, \lambda \in P\left(X_{2}\right), \mu \in M_{1}\left(X_{1}\right)$. We define $(\mu, \lambda, x) \in K$ if there is some $\nu \in P\left(X_{1}\right)$ such that $\lambda=p^{*}(\nu),-\nu \leq \mu \leq \nu$, and $(x, \nu) \in$ $K_{0}$. This is a convex set, which can be realized as a closed convex set in $c_{0}$. Suppose $(\mu, \lambda, x)$ is an extreme point in $K$; clearly, $\mu=\nu$ or $\mu=$ $-\nu$. Thus an extreme point of $K_{0}$ must take the shape $\left(\nu, p^{*}(\nu), x\right)$ or $\left(-\nu, p^{*}(\nu), x\right)$, where $(\nu, x) \in K_{0}$. These can be extreme only if $(\nu, x)$ is extreme in $K_{0}$. It is easy to see that the extreme points of $K$ are just the elements $\left(\delta_{y}, p^{*}\left(\delta_{y}\right), 0\right),\left(-\delta_{y}, p^{*}\left(\delta_{y}\right), 0\right)$ with $y \in \mathcal{S}$. Since $p(\mathcal{S})=\Sigma$, the elements $\left(0, \delta_{z}, 0\right)$ with $z \in \Sigma$ are in the set $\iota(\operatorname{ex} K)$ as each is the average of two extreme points. Conversely, if $\left(0, \delta_{w}, 0\right)$ is in $\iota(\operatorname{ex~} K)$, it is the resultant of an integral over certain elements $(\mu, \lambda, x)$. In this integral $\lambda=\delta_{w}$ a.e., and so, in view of the nature of ex $K_{0}, w \in \Sigma$. Thus $\left(0, \delta_{w}, 0\right)$ is in $\iota\left(\operatorname{ex} K_{0}\right)$ if and only if $w \in \Sigma$, and this is the meaning of Theorem 2 .

Introducing the interval $-\nu \leq \mu \leq \nu$, and averaging measures of opposite sign, allow us to "forget" the measures $\nu$, thus increasing the complexity by one degree. This idea can be traced back to Jayne and Rogers [9], where it is used to pass from Borel sets to co-analytic sets.

Because there exist universal PCA sets $\Sigma$, when we have Theorem 2 for such a set, it follows for all PCA sets at once. 
Representing PCA sets. It will be convenient to have at hand a representation of PCA sets. The main notion is this: A real function $u$ on a product set $A \times B$ is of type $\mathcal{A}_{1}$ if there is some $a \in A$ such that $u(a, b) \neq 0$ for every $b \in B$; otherwise $u$ is of type $\mathcal{A}_{0}$. Let $X$ be an uncountable Polish space and $F$ the metric space of increasing sequences of natural numbers.

(R) Let $S$ be a PCA set in a Polish space $M$. Then there is a uniformly continuous function $u$ to $[0,1]$ on $X \times F \times M$ such that the partial function $u(\cdot, \cdot, m)$ is of type $\mathcal{A}_{1}$ if and only if $m \in \mathcal{S}$.

To define $u$ we begin with a co-analytic set $\mathcal{S}_{1}$ in $X \times M$ whose projection on $M$ is $\mathcal{S}$. Then $(X \times M) \backslash \mathcal{S}_{1}$ is analytic, and is therefore the image $\psi(F)$ of a continuous map $\psi$ on $F$. We write $\psi=\left(\psi_{1}, \psi_{2}\right)$ so that $\psi_{1}(F) \subseteq X$, $\psi_{2}(F) \subseteq M$. Let $\Gamma$ be the set $\left\{\left(\psi_{1}(s), s, \psi_{2}(s)\right): s \in F\right\}$, let $v$ be the function which is distance to $\Gamma$ (any metric can be used), and $u=v /(1+v)$. Since $\psi$ is continuous, $u(x, s, m)=0 \Leftrightarrow x=\psi_{1}(s), m=\psi_{2}(s)$.

To verify that $u$ has the necessary properties, we suppose that $m_{0} \in \mathcal{S}$. Then there is an $x_{0}$ in $X$ such that $\left(x_{0}, m_{0}\right) \in \mathcal{S}_{1}$. For every $s$ in $F$, $\left(\psi_{1}(s), \psi_{2}(s)\right) \neq\left(x_{0}, m_{0}\right)$, so that $u\left(x_{0}, s, m_{0}\right)>0$. Thus the partial function $u\left(\cdot, \cdot, m_{0}\right)$ is of type $\mathcal{A}_{1}$. Conversely, if $m_{1} \notin \mathcal{S}$, then for every $x_{1}$ in $X$, $\left(x_{1}, m_{1}\right) \notin \mathcal{S}_{1}$. Thus there is an $s$ in $F$ such that $x_{1}=\psi_{1}(s), m_{1}=\psi_{2}(s)$, and thus $\psi\left(x_{1}, s, m_{1}\right)=0$. That is, the partial function $u\left(\cdot, \cdot, m_{1}\right)$ is not of type $\mathcal{A}_{1}$.

We use (R) in the case when $X$ is a symmetric set in a Banach space, i.e. $X=-X$; in fact, $X$ is the sphere in a space of dimension at least 2 . We can define $\widetilde{u}$ on $X \times F \times M$ so that it is even with respect to $X$, i.e. $\widetilde{u}(x, s, m)=$ $\widetilde{u}(-x, s, m)$. To attain this we add to $\mathcal{S}_{1}$ the set obtained from it by the map $(x, m) \mapsto(-x, m)$, which is co-analytic and has the same projection. We define $u$ as before, and take finally $\widetilde{u}=\min (u(x, s, m), u(-x, s, m))$. The main point of the variant is this: when $m_{0} \in \mathcal{S}$, then there is some $x_{0}$ such that $\left(x_{0}, m\right)$ and $\left(-x_{0}, m\right)$ belong to $\mathcal{S}_{1}$. Then $u\left(x_{0}, s, m_{0}\right)>0$ and $u\left(-x_{0}, s, m_{0}\right)>0$ for every $s$, i.e. $\widetilde{u}\left(x_{0}, s, m_{0}\right)>0$ for every $s$.

II. Norms and extreme points. Let $X$ be a separable B-space with norm $|\cdot|$ and $\mathbb{N}(X)$ the set of all norms $\|\cdot\|$ equivalent to $|\cdot|$, i.e. satisfying $c_{1}\|x\| \leq|x| \leq c_{2}\|x\|$ for all $x$, with some constants $0<c_{1} \leq c_{2}<\infty$. Provided with the pointwise (product) topology, $\mathbb{N}(X)$ is not quite a metric space but each set $\left\{p \in \mathbb{N}(X): k^{-1} p(x) \leq|x| \leq k p(x)\right\}$ is a compact metric space, and each set $\{p \in \mathbb{N}(X): p(x) \leq k|x|\}$ is a $\sigma$-compact metric space. (We shall gloss over this quibble.)

An interesting subset of $\mathbb{N}$ is the class $\mathcal{R}$ of rotund (strictly convex) norms; this chapter uses a device from a remarkable theorem of B. Bossard $[3,4,5]$. 
Theorem 3. Let $X$ be separable and infinite-dimensional. Then $\mathcal{R}$ is a true co-analytic subset of $\mathbb{N}(X)$, that is, $\mathcal{R}$ is not a Borel set.

The rotundity property of a norm is just the fact that every element of the unit sphere defined by $\|\cdot\|$, i.e. the set $\{\|x\|=1\}$, is an extreme point of that set. We denote by $\varepsilon_{0}$ those norms such that the unit sphere has at least one extreme point. When $X$ has the Radon-Nikodým property (RNP) then every norm has this property [6] and no other spaces are known with this property.

Theorem 4. The set $\varepsilon_{0}$ is of type PCA in $\mathbb{N}(X)$. When $X=c_{0}$ the following holds: For each set $\mathcal{S}$ of type PCA in a Polish space $M$, there is a continuous map $h$ of $M$ into $\mathbb{N}\left(c_{0}\right)$ such that

(i) $h^{-1}\left(\varepsilon_{0}\right)=\mathcal{S}$,

(ii) the map $h$ is continuous into the uniform topology on $\mathbb{N}(X)$, i.e. the topology of uniform convergence on the unit ball of $|\cdot|$.

The first assertion about $\varepsilon_{0}$ is elementary; to prove it we write $p$ for elements in $\mathbb{N}(X)$. The function $p(x)$ defined on $\mathbb{N}(X) \times X$ is measurable, since the set $\{p(x) \leq a\}$ is of type $F_{\sigma}$ for each real $a$. Thus the subset of $\mathbb{N}(X) \times X \times X$ defined as

$$
\{(p, y, z): p(y+z)=p(y)=p(y-z), z \neq 0\}
$$

is a Borel set. The set of pairs $(p, y)$ such that $p(y)=1$, and $y$ is not an extreme point of the unit ball defined by $p$, is an analytic subset of $\mathbb{N}(X) \times X$. Thus $\varepsilon_{0}$ is the projection on $\mathbb{N}(X)$ of a co-analytic set, whence $\varepsilon_{0}$ is of class PCA.

In the second part of Theorem 4 , the space $c_{0}$ enters in two distinct places, so it seems best to write the details for a space $X=Y \oplus c_{0}$ with $Y$ of infinite dimension. Choosing $Y=c_{0}$ we obtain the assertion for $c_{0}$. Theorem 2 does not depend on the norm $|\cdot|$, and we will assume that the norm of $Y$ is locally uniformly rotund (LUR): whenever $\left(y_{n}\right) \subseteq Y$, $y_{0} \in Y,\left|y_{0}\right|=\left|y_{n}\right|=1$, and $\lim \left|y_{0}+y_{n}\right|=2$, then $\lim y_{n}=y_{0}$. Every separable space can be provided with an LUR norm (Kadec, 1950) [7].

Each element $y_{0}$ in the unit sphere of $Y$ is then strongly exposed: let $f_{0} \in Y^{*}$ be such that $\left|f_{0}\right|=1, f_{0}\left(y_{0}\right)=1$. Every sequence $\left(y_{n}\right)$ in the unit sphere of $Y$ such that $f_{0}\left(y_{n}\right) \rightarrow 1$ must converge to $y_{0}$.

Theorem 4 depends on a certain set in the unit sphere of $c_{0}$ which is homeomorphic to $F$ but shares certain properties of compact sets. Let $E_{0}$, $E\left(n_{1}\right), E\left(n_{1}, n_{2}\right), E\left(n_{1}, n_{2}, n_{3}\right), \ldots$ be disjoint, infinite sets of positive integers, defined for $n_{1} \geq 1, n_{2}>n_{1} \geq 1$, etc. Let $\left(n_{k}\right) \in F$; then $\tau\left(n_{k}\right)$ takes value 
- 1 on the first $n_{1}$ elements of $E_{0}$,

- $2^{-1}$ on the first $n_{2}$ elements of $E\left(n_{1}\right)$,

- $2^{-2}$ on the first $n_{3}$ elements of $E\left(n_{1}, n_{2}\right)$,

-...

- 0 elsewhere.

The map $\tau$ is continuous into the norm topology of $c_{0}$. The set $\tau(F)$ has compact closure in $\mathbb{R}^{\mathbb{N}}$ in the product topology; moreover, the product topology agrees with norm convergence in $\tau(F)$. Let $s_{j}=\left(n_{k}(j)\right)$ be a sequence in $F$ such that each sequence $n_{1}(j), \ldots, n_{k}(j)$ converges as $j \rightarrow \infty$ to a limit $N_{k}$ finite or $\infty$. If $N_{1}=\infty$, then the pointwise limit of $\tau\left(n_{k}(j)\right)$ equals 1 on $E_{0}$, and 0 elsewhere. If $N_{1}<\infty$ and $N_{2}=\infty$ then the limit equals 1 on the first $N_{1}$ elements of $E_{0}, 1 / 2$ on all of $E\left(N_{1}\right)$, and 0 elsewhere, etc. Let $H$ be the closure of $\tau(F)$ in the product topology in $\mathbb{R}^{\mathbb{N}}$, and $H^{*}=H \backslash \tau(F)$. Then $H^{*}$ is a countable set $\left(v_{r}\right)_{r=1}^{\infty}$ such that no sum $\sum c_{r} v_{r}$ belongs to $c_{0}$ unless all $c_{r}=0$, where $\sum\left|c_{r}\right|<\infty$. To explain this, we suppose that $v_{1}=(\infty, \infty, \ldots)$. Then $v_{1}=1$ on the infinite subset $E_{0}$, while all of the remaining elements of $H^{*}$ belong to $c_{0}$ on the set $E_{0}$. Hence $c_{1}=0$. Similarly $(1, \infty, \infty, \ldots)$ equals $2^{-1}$ on the infinite set $E(1)$, while all of the remaining elements of $H^{*}$ belong to $c_{0}$ on $E(1)$; for example, $(2, \infty, \infty, \ldots)$ vanishes on an $E(1)$, etc. The property referred to above (stated pedantically) is proved in

Lemma 2. Suppose $S_{1}, S_{2}, \ldots$ is a decreasing sequence of closed subsets of $\tau(F)$, and suppose that $u \in \overline{\mathrm{co}}\left(S_{r} \cup-S_{r}\right)$ for each $r$. Then $u=\int x \lambda(d x)$, where $\lambda$ is a signed Borel measure, of variation at most 1 , concentrated in $\bigcap_{r=1}^{\infty} S_{r} \equiv S$.

Proof. Let $T_{r}$ be the pointwise closure of $S_{r}$ in $\mathbb{R}^{\mathbb{N}}$. By standard limit theorems in measure theory, there is a signed measure $\lambda$, of variation at most 1 , concentrated in $T \equiv \bigcap_{r=1}^{\infty} T_{r}$ such that $u(k)=\int x(k) \lambda(d x)$ for each integer $k=1,2, \ldots$ However, $u$ is an element of $c_{0}$, whence $\lambda$ can have no mass in $H^{*}$. Indeed, the integral of $\lambda$ over $\tau(F)$ is in $c_{0}$, by the remark at the end of this paragraph. The remaining integral is a sum $\sum c_{r} v_{r}$, where $c_{r}$ is the $\lambda$-measure of $v_{r}$, and so each $c_{r}=0$, as explained above. Thus $\lambda$ is concentrated in $T_{r} \cap \tau(F)$ for each $r$, that is, in $S_{r}$, and this proves the lemma. It is worthwhile remarking that every integral $\int x d \lambda(x)$ over $\tau(F)$ is a Bochner (strong) integral, so the sum is in $\overline{\mathrm{co}}(\tau(F) \cup-\tau(F))$.

Let $\theta$ be a uniformly continuous map of $S^{1}(Y) \times F$ into [0,1] which is even with respect to the first element, and let $\|\cdot\|_{\theta}$ be the norm on $Y \oplus c_{0}$ whose unit ball is the closed convex hull of the set $S(\theta):=S^{1}(Y) \cup S^{1}\left(c_{0}\right) \cup$ $\left\{ \pm \theta(y, s) y \pm \tau(s): y \in S^{1}(Y), s \in F\right\}$.

Lemma 3. Let $y_{0} \in S^{1}(Y), x_{0} \in c_{0}, x_{0} \neq 0$. If $\left\|\left(y_{0}, x_{0}\right)\right\|_{\theta} \leq 1$, then $x_{0}$ is in the closed convex hull of the set $\left\{ \pm \tau(s): \theta\left(y_{0}, s\right)=1\right\}$. 
Proof. We apply Lemma 2 to the sequence of closed sets $F_{r}=\{\tau(s)$ : $\left.\theta\left(y_{0}, s\right) \geq 1-r^{-1}\right\}$. Let $f_{0}$ be the bounded linear function on $Y$ which exposes $y_{0}$ strongly, and $g_{0}$ a bounded linear functional on $c_{0}$ such that $g_{0}\left(x_{0}\right)=1$. Let $m$ be a natural number such that $m>\left\|g_{0}\right\|$. The linear functional $f_{0}+m^{-1} g_{0}$ takes the value $1+m^{-1}$ at $\left(y_{0}, x_{0}\right)$, but its values on $S^{1}(Y)$ are at most 1 , and on $S^{1}\left(c_{0}\right)$ less than 1 . Hence it attains a value at least $1+m^{-1}-m^{-2}$ on the third part of the set $S(\theta)$, i.e. at some element $\pm \theta\left(y_{m}, s_{m}\right) y_{m} \pm \tau\left(s_{m}\right)$, with $\left|y_{m}\right| \leq 1$ and $s_{m} \in F$. Then $\left|\theta\left(y_{m}, s_{m}\right)\right| \geq$ $1-m^{-1},\left|f_{0}\left(y_{m}\right)\right| \geq 1-m^{-2}$, and $m^{-1}\left|g_{0}\left(\tau\left(s_{m}\right)\right)\right| \geq m^{-1}-m^{-2}$. As $m \rightarrow \infty$, there is a choice of signs so that $\varepsilon_{m} y_{m} \rightarrow y_{0}$, and then $\theta\left(y_{0}, s_{m}\right) \rightarrow 1$, since $\theta$ is even and uniformly continuous. For large $m, \theta\left(y_{0}, s_{m}\right) \geq 1-r^{-1}$ and $\left|g_{0}\left(\tau\left(s_{m}\right)\right)\right| \geq 1-m^{-1}$. Thus $x_{0}$ is in the closed convex hull of $\pm F_{r}$ for every $r \geq 1$, and we can apply Lemma 2 .

Thus the set mentioned at the conclusion of Lemma 3 is not empty. (Clearly, no conclusions can be drawn if $x_{0}=0$.)

Let $X$ be the space of all sequences $x=\left(y, u_{1}, u_{2}, \ldots\right)$ with $y \in Y$, $u_{n} \in c_{0}$, and $\lim u_{n}=0$. The norm is $\sup \left\|\left(y, u_{n}\right)\right\|_{\theta} \equiv\|x\|_{\theta}$, and clearly $X$ is isomorphic to $Y \oplus c_{0}$.

Lemma 4. (a) Suppose there is some $y_{0} \in S^{1}(Y)$ such that $\theta\left(y_{0}, s\right)<1$ for each $s \in F$. Then $y_{0}=\left(y_{0}, 0,0, \ldots\right)$ is extreme in the unit ball of $X$.

(b) If no element $y_{0}$ of $S^{1}(Y)$ has the property defined in (a), then the unit ball of $X$ has no extreme points.

Proof. (a) Suppose that $y_{0}$ is an average of $\left(y_{1}, u_{1}, u_{2}, \ldots\right)$ and $\left(y_{2},-u_{2}\right.$, $\left.-u_{3}, \ldots\right)$, each of these having norm 1 . Then $\left|y_{1}\right| \leq 1,\left|y_{2}\right| \leq 1,2 y_{0}=y_{1}+y_{2}$. Since the norm of $Y$ is rotund, $y_{1}=y_{2}=y_{0}$. By Lemma 3 , the inequalities $\left\|\left(y_{0}, u_{n}\right)\right\|_{\theta} \leq 1$ imply that each $u_{n}=0$; thus $y_{0}$ is extreme.

(b) Let $x=\left(y_{1}, u_{1}, u_{2}, \ldots\right)$ have norm 1 . If $\left|y_{1}\right|<1$, then $x$ cannot be an extreme point. For we would have $\left|y_{1}\right|+\left|u_{n}\right|<1$ for large $n$, so there would be some $v \neq 0$ in $c_{0}$ such that $\left\|\left(y_{1}, u_{n}+v\right)\right\|_{\theta}<1,\left\|\left(y_{1}, u_{n}-v\right)\right\|_{\theta}<1$. Thus $\left|y_{1}\right|$ must be 1 , and each $u_{n}$ is the resultant $\int \tau(z) d \lambda_{n}(z)$ of an integral over the set $\Sigma$ defined as $\left\{s \in F: \theta\left(y_{1}, s\right)=1\right\}$; the variation of $\lambda_{n}$ is at most 1 . Moreover, since $\Sigma$ is not empty, $\lambda_{n}$ must have variation exactly 1 . The elements $\tau(z)$ of $c_{0}$ have the value 1 at the first member of the set $E(0)$, so that $\left\|\int \tau d \lambda_{n}\right\| \geq\left|\lambda_{n}(F)\right|$.

But this implies that $\lambda_{n}(F) \rightarrow 0$ so that for large $n$ the measures $\lambda_{n}^{+}$ and $\lambda_{n}^{-}$are different from 0 . From this and the inequality on $\int \tau d \lambda_{n}$, we see that $x$ cannot be extreme.

To complete the proof of Theorem 4, we make use of the representation (R) of the previous section, taking for $X$ the unit sphere $S^{1}(Y)$ of $Y$. We map an element $m$ of $M$ to the norm $\|\cdot\|_{\theta}$, where $\theta$ is the partial function $1-u(\cdot, \cdot, m)$ defined on $S^{1}(Y) \times F$. The symmetry of $\theta$ on $S^{1}(Y)$ is obtained 
in a remark to (R), and the continuity in point (ii) follows from the uniform continuity of $u$.

III. Extreme points, redux. Let $X$ be a separable B-space and $\mathcal{E}$ the set of extreme points of its unit ball. Then NA denotes the set of linear functionals that attain their norm on the unit ball, and NAE those that attain their norm on $\mathcal{E}$. When $X^{*}$ is provided with the $w^{*}$-topology, NA is analytic and NAE is a PCA $\left(\Sigma_{2}^{1}\right)$ set.

THEOREM. The space $c_{0}$ can be provided with a norm $|\cdot|$ so that NAE is then a complete PCA set.

Completeness of NAE will be established in the same form as in previous sections, via a map $\varphi$ which is continuous into the norm of $c_{0}^{*}$. We observe that NAE is analytic if $\mathcal{E}$ is a Borel set and also in certain other cases. For if $X$ has the Radon-Nikodým property (RNP), as $\ell^{1}$ clearly has, then $\mathrm{NAE}=\mathrm{NA}$. It seems likely, on the basis of $[9,10,12]$, that $\ell^{1}$ can be normed so that $\mathcal{E}$ is not a Borel set.

We write $\|\cdot\|$ for a norm on $X$, the classical one for $c_{0}$, but in fact this norm plays almost no rôle in the proof. Let $K$ be a closed, bounded, convex set in $X$, let $B$ be the unit ball for the norm $\|\cdot\|$, and let $|\cdot|$ be the norm whose unit ball is $B^{\sim}=\overline{\mathrm{co}}\left(\frac{1}{2} B \cup K \cup-K\right)$. Henceforth $\mathcal{E}$, NA, and NAE refer to this norm. We introduce the following condition on functionals $f$ in $X^{*}$ :

$\left(*^{*} *\right) \quad f \geq 0$ on $K$ and $\sup f(K)>\|f\| / 2$.

Then $f \in \mathrm{NA}$ (for the norm $|\cdot|$ ) if and only if $f$ attains its norm on $K$; and if $f \in \mathrm{NAE}$ then $f$ must attain its norm on ex $K$. Conversely, always subject to $\left(*^{*} *\right)$, if $f$ attains its norm at an element $x_{0}$ of ex $K$ then $x_{0} \in \mathcal{E}$, because $f \leq 0$ on $-K$ and $f \leq\|f\| / 2<f\left(x_{0}\right)$ on $\frac{1}{2} B$. Thus $f \in$ NAE.

Next we summarize the conclusions of $[10,12]$, beginning with a compact metric space $M$, a co-analytic subset $\mathcal{S}$ of $M$, and the convex set $P(M)$ of probability measures. We represent $K$ at first as a closed, bounded, convex subset of $P(M) \oplus B$, where $B$ is the unit ball of $c_{0} ; M$ has the following properties:

(i) $K$ contains the set $P(M) \oplus(0)$.

(ii) The extreme points of $K$ are the elements $\left(\delta_{y}, 0\right)$, with $y \in \mathcal{S}$.

Next we replace $P(M)$ by a representation in $B$ : we map each measure $\mu$ in $P(M)$ to a sequence $L \mu=\left(\left\langle\mu, g_{k}\right\rangle\right)_{k=1}^{\infty}$ where $\left(g_{k}\right)_{k=1}^{\infty}$ is a total sequence in $C(M)$ and $\sup \left|g_{k}\right|=o(1)$. Henceforth we construe $K$ as a convex subset of $c_{0} \oplus c_{0} \sim c_{0}$.

The linear functionals we use in the theorem act on the first factor in $c_{0} \oplus c_{0}$, i.e. on the factor in which $P(M)$ is represented. Suppose $f=\left(b_{k}\right)_{k=1}^{\infty}$ 
is a sequence in $\ell_{1}=c_{0}^{*}$. Its norm as a functional on $c_{0} \oplus c_{0}$ is of course $\sum\left|b_{k}\right|$, whereas its value at the sequence $L \mu$ is $\sum_{k=1}^{\infty} b_{k}\left\langle\mu, g_{k}\right\rangle$. Hence $\left(*_{*}^{*}\right)$ is true provided $\sum b_{k} g_{k} \geq 0$ everywhere in $M$ and $\sup \sum b_{k} g_{k}>\sum\left|b_{k}\right| / 2$. If these conditions are satisfied, then $f \in \mathrm{NAE}$ if and only if $\sum b_{k} g_{k}$ attains its supremum (on $M$ ) in the subset $\mathcal{S}$.

We now specify that $M$ is the circle of length $2 \pi$,

$$
\begin{array}{rlrl}
g_{2 k+1} & =(k+1)^{-1 / 3} \cos k t, & k & =0,1,2, \ldots, \\
g_{2 k} & =(k+1)^{-1 / 3} \sin k t, \quad k & =1,2, \ldots
\end{array}
$$

Then every function $u$ in the class $\operatorname{Lip}^{1}(M)$ admits exactly one expansion $\sum_{k=1}^{\infty} b_{k} g_{k}$ with $\sum\left|b_{k}\right|<\infty$, and therefore there is a functional, written $\alpha(u)$, such that $\langle\alpha(u), L \mu\rangle \equiv \int u d \mu$. These assertions are consequences of Parseval's formula and Cauchy's inequality. In fact, $\|\alpha(u)\| \leq \sup |u|+$ $c$ ess $\sup \left|u^{\prime}\right|$, with a certain constant $c$; a bit more work yields an upper bound $c \delta^{-5} \sup |u|+c \delta$ ess sup $\left|u^{\prime}\right|$, for all $\delta \in(0,1)$.

We can find co-analytic sets $\mathcal{S}$ which can be mapped continuously onto any PCA set, for example the set WF of trees with no infinite branch. Since the set of trees is 0 -dimensional we can place $\mathcal{S}=\mathrm{WF}$ in the $\operatorname{arc}(\pi / 4, \pi / 2)$ of $M$. Let $\Sigma$ be a PCA set in a metric space $N$ of diameter at most 1 , so that $\Sigma=h(\mathcal{S})$, a continuous image of $\mathcal{S}$. We define $F$ on $M \times N$ by

$$
F(t, y)=\inf \{|t-s|+d(h(s), y)): s \in \mathcal{S}\}
$$

when $t \in M, y \in N$. Then $F$ is jointly continuous on $M, 0 \leq F \leq 1+\pi<5$, and $\left|F\left(t_{1}, y\right)-F\left(t_{2}, y\right)\right| \leq\left|t_{1}-t_{2}\right|$. If $y \in \Sigma$ then $F(s, y)=0$ for some $s \in \mathcal{S}$, and the converse is true because $h$ is continuous on $\mathcal{S}$. Since $|\sin s|>1 / 2$ for each $s$ in $\mathcal{S}$, the remark above remains true for the function $G(t, y) \equiv$ $|\sin t| F(t, y)$. Let $\eta>0$ be a small constant. We define a map $\psi$ from $N$ into $\ell^{1}$ as follows. We apply $\alpha$ to the partial function $1-\eta G(\cdot, y)$. Each of these functions on $M$ has supremum 1 and is positive if $0<\eta<1 / 5$. When $\eta$ is small enough, the resulting functional satisfies $\left(*^{*} *\right) ; \psi(y)$ belongs to NAE if and only if $1-\eta G(s, y)=1$ for some $s$ in $\mathcal{S}$, that is, $y \in \Sigma$. The continuity of $\psi$ is a consequence of the refined inequalities written above.

\section{References}

[1] H. Becker, Pointwise limits of sequences and $\Sigma_{2}^{1}$ sets, Fund. Math. 128 (1987), 159-170.

[2] H. Becker, S. Kahane and A. Louveau, Some complete $\Sigma_{2}^{1}$ sets in harmonic analysis, Trans. Amer. Math. Soc. 339 (1993), 323-336.

[3] B. Bossard, Théorie descriptive des ensembles en géométrie des espaces de Banach, thèse, Univ. Paris VII, 199?.

[4] -, Co-analytic families of norms on a separable Banach space, Illinois J. Math. 40 (1996), 162-181. 
[5] B. Bossard, G. Godefroy and R. Kaufman, Hurewicz's theorems and renorming of Banach spaces, J. Funct. Anal. 140 (1996), 142-150.

[6] R. G. Bourgin, Geometric Aspects of Convex Sets with Radon-Nikodym Property, Lecture Notes in Math. 993, Springer, 1983.

[7] R. Deville, G. Godefroy and V. Zizler, Smoothness and Renormings in Banach Spaces, Pitman Monogr. Surveys Pure Appl. Math. 64, Longman Sci. Tech., 1993.

[8] G. A. Edgar, A noncompact Choquet theorem, Proc. Amer. Math. Soc. 49 (1975), 354-358.

[9] J. E. Jayne and C. A. Rogers, The extremal structure of convex sets, J. Funct. Anal. 26 (1977), 251-288.

[10] R. Kaufman, Co-analytic sets and extreme points, Bull. London Math. Soc. 19 (1987), 72-74.

[11] —, Topics on analytic sets, Fund. Math. 139 (1991), 217-229.

[12] -, Extreme points and descriptive sets, ibid. 143 (1993), 179-181.

[13] R. R. Phelps, Lectures on Choquet's Theorem, Van Nostrand Math. Stud. 7, Van Nostrand, Princeton, NJ, 1966.

Department of Mathematics

University of Illinois

1409 West Green Street

Urbana, IL 61801, U.S.A.

E-mail: rpkaufma@math.uiuc.edu

Received 4 May 1999;

in revised form 29 November 1999 